\title{
Identity and density of parasite exposures alter the outcome of co-infections
}

\author{
Chloe Ramsay ${ }^{1}$ and Jason Rohr ${ }^{1}$ \\ ${ }^{1}$ University of Notre Dame
}

May 5, 2020

\begin{abstract}
Although research has focused on density-dependent responses to single parasite infections, much less in known about how parasite density affects the more common scenario in nature, co-infections. We investigate how parasite density alters co-infection dynamics by simultaneously exposing Cuban treefrogs (Osteopilus septentrionalis), in all pairwise combinations and at a range of doses, to: the nematode Aplectana hamatospicula, the chytrid fungus (Batrachochytrium dendrobatidis), and Ranavirus. The latter two parasites are implicated in widespread amphibian declines. We found that all pairwise co-infections were density dependent, but some were positively and others negatively density dependent, and these effects drove host pathology. Also, all co-infections were highly asymmetric - strong in one direction and weak in the other - consistent with weak and asymmetric interactions dominating food webs and mutualistic networks. These findings suggest that the null expectation for co-infections should be that they are density-dependent, asymmetric, and important to host health.
\end{abstract}

\section{Introduction:}

Co-infections, where hosts are infected with more than one parasite at a time, are extremely common (Greub et al. 2000; Kim et al.2003; Swanson et al. 2006). Co-infections can be important because they can cause variation in disease progression that can have significant negative or positive effects on the health of hosts by altering resistance (i.e ., ability to reduce the abundance of parasites) or tolerance (i.e ., ability to reduce the per capita cost of parasites to maintain health) to parasites. For example, HIV infection reduced resistance to and mortality by a Mycobacterium that causes tuberculosis in humans (Corbett et al. 2003), whereas coinfection with helminths improved resistance and reduced the spread of bovine tuberculosis in African buffalo populations (Ezenwa \& Jolles 2015). Mice co-infected with influenza virus and Legionella pneumophila were less tolerant of tissue damage associated with infection than singly infected mice (Jamieson et al. 2013).

Co-infecting parasites can interact directly or indirectly within hosts (Kuris \& Lafferty 1994; de Roode et al. 2004). For parasites to interact directly, they typically infect the same tissue of hosts or use the same resources. This is common when parasites are closely related to each other. For example, when testing genetically divergentPlasmodium chabaudi clones, strong competition between the strains was predicted by strain identity (Bell et al. 2006). Indirect interactions between parasites are often mediated by the host immune system. These indirect interactions thus can play a role in disease progression even if the parasites are unlikely to ever come into contact within the host. For example, macroparasites activate the T helper 2 (Th2) arm of the immune system (Paul \& Zhu 2010), microparasites activate the Th1 arm of the immune system, and fungal infections can activate the Th17 arm (Berger 2000; Blanco \& Garcia 2008; Zelante et al. 2009). If two parasites activate the same portion of the host immune system (e.g. co-infection with two microparasites), they have the potential to inhibit one another by activating immune responses that combat both parasites, which is often referred to as cross-immunity (Johnson \& Buller 2011). If two parasites activate different branches of the host immune system, they have the potential to facilitate one another. This is because the Th1 and Th2 arms limit one another and therefore hosts have trouble upregulating both 
simultaneously (Abbas et al. 1996; Morel \& Oriss 1998; Yazdanbakhsh et al. 2002). As an example, mice infected with Toxoplama gondii mounted a Th1 response and it limited the host's ability to mount a Th2 response against subsequent Heligmosomoides polygyrus infection (Ahmed et al. 2017).

Because co-infecting parasites interact with one another and the environment of the host, community ecology principles, such as negative density dependence, may alter host health and inform our understanding of coinfections (Pedersen \& Fenton 2007; Johnson et al. 2015). Negative density dependence predicts that the strength of competition between species can depend on their densities, with potential impacts for community assembly (Chesson \& Kuang 2008). Hence, all else being equal between species, the species at the higher density should outcompete the one at the lower density. Although there are many studies on the effects of parasite density or dose on single-species infections (Khuroo 1996; Pearman et al. 2004; Garner et al. 2009; Echaubard et al. 2010), few studies have examined how parasite density or dose alters co-infections and associated disease progression (Ge et al. 2012; Niczyporuk et al. 2014). The terms density and dose will be used interchangeably throughout the remainder of the manuscript. Of the few studies that have considered this, they have either focused on closely related parasite taxa (Ge et al.2012) or included other variables in their design, such as vaccination, making it difficult to parse the effects of density of co-infecting parasites on disease progression (Niczyporuk et al. 2014). Hosts are often concomitantly exposed to multiple parasites in nature, and due to the spatiotemporal variation in abundance of parasites that a host might encounter, co-infection with multiple parasites at different densities is more likely in the wild than co-infections with identical parasites densities. Thus, our experiment aims to test how co-infection with varying doses alters the outcome of co-infections and disease in amphibians.

Amphibians are the most highly threatened vertebrate taxa partly due to the spread of parasites (Wake \& Vredenburg 2008). To address this aim, we exposed Cuban treefrogs (Osteopilus septentrionalis) to pairwise co-infections of the following parasite species:Batrachochytrium dendrobatidis (Bd), Ranavirus, and Aplectana hamatospicula. Importantly, we tested how the dose of each species affected co-infection and disease progression. Bd is a chytrid fungus that is associated with extinctions and population declines of amphibians globally (Berger et al. 1998; Skerrattet al. 2007; Kilpatrick et al. 2010). The infectious zoospores of this parasite enter the skin of amphibians and fuse the keratinous portions of the skin (Kilpatrick et al. 2010). This limits osmoregulation and can cause cardiac arrest (Voyles et al.2009). Ranavirus is a virus that causes mass mortality in amphibians also on a global scale (Green et al. 2002; Gray et al.2009). Ranavirus is from the family Iridoviridae and it replicates in the internal organs of ectothermic vertebrates, causing hemorrhaging of these organs, particularly in the liver and kidneys (Gantress et al. 2003; Gray et al. 2009). These two parasites are microparasites, meaning that they are small and that they can replicate internally in the host, leading to higher parasite loads even without repeated exposure (Rojas et al. 2005; Rosenblumet al. 2012). Alternatively, we tested a macroparasite, the nematode A. hamatospicula, which is a common parasite of amphibians in the Southeastern US and Latin America (Baker 1987; Vhora \& Bolek 2013; Ortega et al. 2015). A. hamatospiculainfects the host when juveniles penetrate the skin and migrate to the amphibian gastrointestinal tract (Knutie et al. 2017b). There, they mature into adults eventually passing juveniles through the feces that can re-infect the frogs (Knutie et al. 2017b). Parasitic worms, Ranavirus, and Bd frequently co-occur in amphibian hosts in the wild (Hoverman et al. 2012; Stutz et al. 2018; Watterset al. 2018). In fact, in a biodiversity hotspot in the Peruvian Andes, up to $50 \%$ of frogs were co-infected with Ranavirus and Bd (Warneet al. 2016).

The goals for this study were to test if the dose of parasite exposure alters 1) the load of the co-infecting parasite (i.e ., resistance), 2) survival or growth rate of the host, and 3) the host's ability to tolerate infections. These goals were motivated by several hypotheses. First, we hypothesized that the load of one infecting parasite would be negatively associated with the exposure dose of the co-infecting parasite because of density-dependent competition. We also hypothesized that the advantage a parasite has when it is at a high density would be amplified if the co-infecting parasite activates a similar immune response (i.e., are both microparasites) and lessened or potentially facilitated if the co-infecting parasite activates a different immune response (i.e., a micro- and microparasite). Second, we hypothesize that co-infection will decrease a host's tolerance (e.g. a host's ability to maintain their health despite high infection loads) when the 
co-infecting species are facilitative and increase host tolerance when they inhibit one another.

\section{Methods:}

\section{Animal Husbandry}

Cuban treefrog tadpoles (Osteopilus septentrionalis ) were collected from kiddy pools (140 L) filled with water in the University of South Florida Botanical Gardens (Tampa, FL, USA) in August 2016. Individuals were raised in plastic containers filled to $11 \mathrm{~L}$ with artificial spring water (ASW) at densities of 20-30 tadpoles/container and were fed ad libitum every other day a mixture crushed fish flakes (United Pet Group, Inc., Blacksburg, VA, USA), spirulina (NOW foods, Bloomingdale, IL, USA), in an agar block (Fisher BioReagents, Pittsburgh, PA, USA). Tadpoles were moved to new containers with fresh ASW weekly. Used plastic containers were washed by hand, bleached, rinsed with deionized (DI) water, and dried weekly. Once tadpoles grew back legs lids were placed on the container to prevent escape. When metamorphosing frogs grew front legs, frogs were housed in $57.75 \mathrm{~cm}^{3}$ sterilized plastic cups containing an unbleached paper towel and $10 \mathrm{~mL}$ of ASW. After metamorphosis frogs were held in sterilized plastic cups containing an unbleached paper towel wet with ASW and frogs were fed 2-week-old crickets ad libitum twice a week. Containers were also changed weekly and cleaned as described above. All frogs were housed at $25^{\circ} \mathrm{C}$ with a $12 \mathrm{~h}$ photoperiod.

Experimental Design

To examine how the dose of parasite exposures affects host-parasite dynamics, we exposed frogs to one of 28 total parasite treatments. Treatments included exposure to Ranavirus alone, $\mathrm{Bd}$ alone, A. hamatospicula alone, all pairwise combinations of these parasites at a range of densities, and controls (no exposure). In each of the pairwise co-infection treatments hosts were exposed to one parasite at an intermediate density and the second parasite at one of a range of densities (one of four potential densities) in all possible combinations (Table 1). There were six individuals for each treatment group $(n=6)$, and twelve control individuals $(n=12$ ), for a total of 174 frogs.

Parasite culture

A. hamatospicula were collected from the gastrointestinal tract of euthanized Cuban treefrogs. Cuban treefrogs were collected from Flatwoods Wilderness Park (Tampa, FL, USA). After extraction, worms were stored in Petri dishes $(15 \times 150 \mathrm{~mm} ; n$ [?] 5 per dish) filled with $2 \mathrm{~mL}$ of DI water and gastrointestinal content from the infected amphibians in dark conditions. After the adult female worms laid larval worms (J1 larvae), they were kept for at least 14 days to ensure that larval worms reached the infectious stage (J3 larvae). Petri dishes with $2 \mathrm{~mL}$ of DI water and gastrointestinal content from uninfected frogs collected to act as a sham treatment.

Ranavirus (FV3) was cultured in fathead minnow (Pimephales promelas ) cells and maintained at -80degC in minimal essential medium (MEM).

Bd (SRS-JEL 212 strain) in a $1 \%$ tryptone solution was aliquoted into Petri dishes previously filled with $1 \%$ agar and $1 \%$ tryptone gel. $\mathrm{Bd}$ was grown at $23 \mathrm{degC}$ for seven days until zoosporangia had matured and were releasing zoospores, after which, $1 \mathrm{~mL}$ of DI water was added to the Petri dishes and gently mixed to encourage zoospore release. To asses Bd zoospore concentration, we poured this solution into a beaker and diluted it until we counted a final Bd density of $10^{5}$ zoospores $/ \mathrm{mL}$ using a hemocytometer. Plates with a sterile $1 \%$ tryptone solution without Bd were stored and washed identically to the Bd plates to produce sham treatments.

Parasite exposures

Two days before parasite exposure, individuals were moved to an environmental chamber kept at $17 \mathrm{deg}$ C because Cuban treefrogs can clear Bd at higher temperatures (McMahon et al. 2014; Cohen et al.2017). In the co-infection treatments, the host was exposed to one parasite at an intermediate, fixed dose and to the other parasite at a dose that ranged from low to high for each of the tested parasites (Garner et al. 2009; Echaubard et al. 2010; Hovermanet al. 2010; Gervasi et al. 2013; Knutie et al.2017a; Knutie et al. 2017b). 
For the microparasites $\mathrm{Bd}$ and Ranavirus, doses ranged from $10^{3}$ to $10^{5}$ zoospores or plaque forming units (PFU), respectively, and the intermediate dose was $10^{4}$ (Table 1). For the macroparasite A. hamatospicula , doses ranged from 15 to $60 \mathrm{~J} 3$ larvae and the intermediate dose was 30 (Table 1). These doses were applied to frogs held in Petri dishes $(25 \times 100 \mathrm{~mm})$. Frogs received the doses for their randomly assigned treatment as 1-mL of DI water with Bd zoospores, or with J3 A. hamatospicula, or a $69 \mu$ l aliquot of MEM with Ranavirus. All hosts also received sham exposures for parasites to which they were not exposed to ensure that each frog received identical treatments with the exception of the parasite. For example, hosts co-exposed to $\mathrm{Bd}$ and $A$. hamatospicula, received a $69 \mu \mathrm{l}$ aliquot of MEM without Ranavirus. Control individuals received sham treatments for all three parasites.

Amphibian responses to parasites

To measure amphibian growth, frogs were weighed weekly for four weeks. Individuals were also checked twice daily for mortality. If mortality occurred, frogs were weighed and swabbed for Bd and/or Ranavirus and/or dissected to assess A. hamatospicula loads in their gastrointestinal tract, depending on their treatment group. All surviving frogs were euthanized and dissected 28 days after initial parasite exposure. Growth rates were calculated as the final mass minus the initial mass divided by weeks spent alive $\left(\mathrm{g} \mathrm{wk}^{-1}\right)$

Assessing parasite loads

To assess loads of Ranavirus, hosts were swabbed five times around the mouth and cloaca on days 2, 4, and 8. To assess Bd load, hosts were swabbed five times from hip to toe on both rear legs on days 8, 16, and 24 after exposure. Swabs were placed in $2 \mathrm{~mL}$ sterile microcentrifuge tubes and stored at $-80^{\circ} \mathrm{C}$ for later processing. A. hamatospicula loads were assessed by checking the frog's feces weekly for larval worms and dissecting individuals and checking their gastrointestinal tract for adult worms either when they experienced mortality or at the end of the experiment.

DNA from each swab was extracted using a Qiagen DNEasy Blood \& Tissue Kit and analyzed using quantitative polymerase chain reaction (qPCR; Boyle et al. 2004; Picco et al. 2007). Days 16 and 4, for Bd and Ranavirus respectively, were chosen for statistical analyses after initial qPCR analyses, where we analyzed subsets of swabs across treatments across days. From these initial analyses these days were chosen as optimal time points for assessing load differences as they were both early enough for individuals to not have experienced mortality from the infections, but also late enough to address parasite establishment (Gray et al. 2009; Voyles et al. 2009).

\section{Statistical Analyses}

All analyses were run with $\mathrm{R}$ version 3.6.1 (R Core Team 2019). Plots were created using the visreg package and visreg function (Breheny \& Burchett 2019). Survival plots were created using thesurvminer package and ggsurvplot function (Kassambaraet al. 2019).

How do changing doses of parasite affect the resistance to the fixed dose, co-infecting parasite?

To test how the interaction between the identity of a co-infecting parasite and its dose alter the load of Ranavirus or Bd, we conducted a generalized linear model (glm) with a negative binomial error distribution. For A. hamatospicula load, we used logistic regression ( $g l m$ function) with a binomial error distribution, where the dependent variable was defined as the number of larvae that successfully infected (penetrated the host, migrated to gut, and reached maturity) over the total number of larvae to which the hosts were exposed. The independent variables for all of the above models were the crossed dose and identity of the changing-dose parasite. Dose was expressed as a proportion of the highest possible dose hosts were exposed to for each parasite. Altering dose to be a proportion allowed us to make comparisons across doses even though the quantity of dose we exposed hosts to varied widely for macro- and microparasites. For this and all further described analyses Tukey Post-hoc tests were run to compare among parasite identities when the main effect or interaction were significant (multcomp package and glht function (Hothorn 2010).

How do parasite dose and identity affect host growth, survival, and tolerance? 
To test how the interaction between the identity of a co-infecting parasite and its dose affect host growth we conducted general linear models. To address change in host weight, growth rate was calculated as weight of the host at the end of experiment or on the day of death divided by the total weeks alive (g/week) and used as the dependent variable. The identity of the fixed dose parasite, the identity of the changing dose parasite, and the dose of the changing dose parasite were used as crossed independent variables. To test how these same factors affected tolerance based on growth, this same model was repeated with the addition of parasite load of the fixed-dose parasite as a third, crossed independent variable.

To address how the identity of the co-infecting parasites and dose of exposure affected host survival, we conducted a survival analysis (using the survival package and the coxph function (Therneau \& Lumley 2019) with host survival as the dependent variable. The identity of the fixed- and changing-dose parasites, as well as the dose of the changing-dose parasite, were crossed independent variables. To test how these same factors affected tolerance based on survival, this same model was repeated with the addition of parasite load of the fixed-dose parasite as a third, crossed independent variable.

\section{Results}

How do changing doses of parasites affect resistance to the fixed dose, co-infecting parasite?

Analyses revealed a significant negative association between the dose of the co-infecting parasite and the load of Ranavirus, regardless of the identity of the co-infecting parasite $\left(\chi^{2}{ }_{1}=4.07, p=0.04\right.$; Fig. 1$)$. For $A$. hamatospicula, there was an interaction between the identity and the dose of the changing-dose parasite $\left(\chi^{2}{ }_{1}=12.42, p<0.001\right)$. Dose of Bd was associated negatively with A. hamatospicula $(p<0.001$; Fig. 2$)$, whereas dose of Ranavirus was not significantly associated with A. hamatospicula load ( $p=0.22$; Fig. 2).

Neither the identity of the co-infecting parasite $\left(\chi^{2}{ }_{1}=0.03, p=0.86\right)$ nor its dose $\left(\chi^{2}{ }_{1}=0.01, p=0.92\right)$ affected Bd load in co-infected hosts.

How do parasite dose and identity affect host growth, survival, and tolerance?

The identity of both fixed $\left(F_{2,157}=35.5, p<0.001\right)$ and changing dose parasites $\left(F_{2,157}=10.89, p<0.001\right)$ were significant predictors of weight change. For both the fixed- and changing-dose parasites, growth was significantly higher for control hosts than infected hosts (controls vs. Bd: fixed dose parasite and changing dose parasite: $p<0.001$; controls vs. A. hamatospicula : fixed dose parasite: $p=0.02$, changing dose parasite: $p=0.01$; controls vs. Ranavirus: fixed dose parasite: $p=0.01$, changing dose parasite: $p<0.01$ ). Additionally, exposure to $\mathrm{Bd}$ at a fixed dose caused a significant decrease in host growth rate ( $\mathrm{g} /$ week) when compared to exposure to A. hamatospicula (Bd vs. A. hamatospicula : $\mathrm{p}<0.001$ ) or Ranavirus (Bd vs. Ranavirus: $\mathrm{p}<0.001$ ). Dose of co-infecting parasite was not a significant predictor of weight gain when all parasites were analyzed together $\left(F_{1,157}=1.75, p=0.19\right)$, but dose of Bd was negatively associated with host growth $\left(F_{1,46}=8.8, p\right.$ $<0.005)$.

The identity of the fixed-dose parasite was the only significant predictor of host survival $\left(F_{2,125}=17.03, p\right.$ $<0.001$ ), with exposure to Bd significantly reducing host survival relative to Ranavirus (Bd vs. Ranavirus: $p$ $<0.001$ ), A. hamatospicula (Bd vs. A. hamatospicula : $p<0.001$ ), and the uninfected controls (Bd vs. control: $p<0.001 ;$ Fig.4).

We found no significant effects of dose or identity of the co-infecting parasite on host tolerance of Ranavirus (Viral load $\mathrm{x}$ dose: $F_{1,37}=0.02, p=0.9$; Viral load $\mathrm{x}$ identity of co-infecting parasite: $F_{2,37}=1.89, p=0.17$ ) or A. hamatospicula (A. hamatospicula loadx dose: $F_{1,39}=0.49, p=0.49 ;$ A. hamatospicula load $\mathrm{x}$ identity of co-infecting parasite: $F_{1,39}=0.02, p=0.88$ ).

However, dose and identity of the co-infecting parasite interacted to affect host tolerance of $\mathrm{Bd}$ ( $\mathrm{Bd}$ load $\mathrm{x}$ dose $\mathrm{x}$ identity: $F_{1,44}=6.0, p=0.02$ ). Tolerance (defined as less negative slopes between parasite load and weight gain) of $\mathrm{Bd}$ based on host weight gain was associated positively with the dose of A. hamatospicula, but negatively with the dose of Ranavirus (Fig. 5). We found no significant effects of host tolerance when using host survival as a metric for tolerance. 


\section{Discussion:}

Hosts are frequently co-infected and there is spatiotemporal variation in the densities of parasite that they encounter. We found that every set of pairwise co-infections was density dependent. Additionally, each was highly asymmetric and weak in one of the two directions, consistent with other ecological interactions, such as the asymmetric and weak interactions that dominate food webs (Paine 1992; McCann et al.1998) and mutualistic networks (Bascompte et al. 2006). We also found that the density of a co-infecting parasite has the potential to alter the loads of the other co-infecting parasites and host tolerance these infections.

In co-infections with Ranavirus and A. hamatospicula, higher doses of A. hamatospicula lowered viral load (Fig. 1), but higher doses of Ranavirus did not alter A. hamatospicula load (Fig. 2). The negative associations between $A$. hamatospicula and Ranaviral load are inconsistent with our hypothesis of positive density dependent competition between macro- and microparasites. Given that viruses elicit the Th1 arm of the immune system, whereas helminths upregulate the Th2 arm and these two arms are well-documented to be antagonistic in vertebrate host taxa (Degen et al. 2005; Graham 2008; Salgameet al. 2013), the expectation was that co-infection with Ranavirus and A. hamatospicula would be facilitative rather than inhibitory. When the dose of Ranavirus was manipulated, there was a non-significant trend in support of facilitation of $A$. hamatospicula that was consistent with the hypothesized positive density dependence. In contrast, however, when A. hamatospiculawas manipulated, there was significant inhibition of Ranavirus or significant negative density dependence. This competition is likely immune-mediated because these parasites are unlikely to interact directly given that Ranavirus infects the liver and kidneys (Grayet al. 2009) and A. hamatospicula lives in the gastrointestinal tract (Knutie et al. 2017b). Additionally, the host immune response to these parasites might be further complicated by the fact that A. hamatospicula, in entering through the skin of the amphibians, has to combat the complex chemical and physical defenses present in the mucosa of amphibians (reviewed in Fites et al.2014) and Ranavirus does not when it is ingested, the method we used for this experiment. Therefore, either this polarization between the Th1 and Th2 immune system does not differentiate as strongly because the mucosal immune system combats parasites before they even enter the amphibians or there is another mechanism responsible for this response.

In co-infections with Ranavirus and Bd, higher doses of Bd decreased viral load (Fig. 1), but higher doses of Ranavirus did not impact Bd load. We hypothesized negative density dependence between these parasites because they are both microparasites that activate similar arms of the host immune system (Berger 2000). We found evidence to support this hypothesis, but only in one direction. Ranavirus was outcompeted at higher Bd loads. Bd and Ranavirus are unlikely to interact directly in the host because Bd infects the skin of amphibians (Berger et al. 2005) and Ranavirus infects the liver and kidneys (Gray et al. 2009), but results show that there is the potential for Bd to inhibit Ranavirus infection through cross-immunity (Berger 2000). Interestingly, this pattern does not occur in the reverse direction, suggesting that the immune response to combat Bd can successfully combat Ranavirus, but the immune response to combat Ranavirus cannot successfully combat Bd. Although our understanding of whether amphibians can upregulate a Th17 immune response is limited (Fites et al. 2014), Th17 is frequently upregulated in conjunction with Th1 immune responses to combat fungal infections in other vertebrates (Blanco \& Garcia 2008). Therefore, this difference in how host immune systems respond to Bd versus Ranavirus could make our hypothesis of cross-immunity between these parasites overly simplistic. Additionally, we found that our hosts are more susceptible to Bd infection than the other parasites tested (Fig. 3, Fig. 4). Therefore, if Bd mounts a mix of a Th1 and Th17 immune responses, the mounted Th1 response may function through cross-immunity to combat Ranavirus, but if Ranavirus only mounts a Th1 response, that might not be a strong or complex enough immune response to successfully combat Bd.

In co-infections with $\mathrm{Bd}$ and $A$. hamatospicula, the dose of $\mathrm{Bd}$ negatively impacted the load of $A$. hamatospicula (Fig. 2), but the dose of A. hamatospicula did not alter the load of Bd. We hypothesized positive density dependence for this interaction because $\mathrm{Bd}$ and $A$. hamatospicula likely activate different parts of the host immune system, with Bd activating the Th1 and Th17 arms, although the use of Th1 and/or Th17 to combat Bd is not fully characterized (Fites 2014), and A. hamatospicula activating the Th2 arm (Berger 
2000; Paul \& Zhu 2010). We infected hosts simultaneously, so Bd would not be established in the skin when A. hamatospicula infects and therefore it is unlikely that this direct interaction plays a role in competitions between these parasites (Berger et al. 2005; Knutieet al. 2017b). Therefore, interactions between parasites are likely to be indirect and mediated by the host immune system. Although not originally hypothesized, there is the potential that this interaction could be largely driven by innate, instead of acquired, immunity. For example, macrophages can combat both Bd (Fites 2014) and helminth infections (Motran et al. 2018) and short-term upregulation of innate immune responses (i.e. macrophages) could be driving the decrease in $A$. hamatospciula load at higher $\mathrm{Bd}$ doses, especially if macrophages are more effective against $A$. hamatsopicula than Bd.

Host tolerance to Bd was significantly impacted by an interaction between identity and dose of the coinfecting parasites. Tolerance (defined as less negative slopes between parasite load and weight gain) of Bd was associated positively with the dose of A. hamatospiculabut negatively with the dose of Ranavirus (Fig. 5 ). This pattern was not in keeping with our hypotheses that microparasites would upregulate similar arms of the host immune system and therefore inhibit each other, and co-infections with macro- and micro-parasites would facilitate one another through tradeoffs in immune activation, causing increases and decreases in host tolerance, respectively. In fact, we saw the opposite pattern. Additionally, we found that exposure to Bd significantly decreased host growth and survival relative to controls and more so than any other parasite (Fig.3, Fig.4). This suggests that Cuban treefrogs are particularly susceptible to Bd infection. Infection with Ranavirus and A. hamatospicula also decreased host growth when compared to individuals without infections (Fig. 3). Therefore, the fact we only found significant results on amphibian tolerance to Bd is in keeping with our other results on host health. Our results suggest that hosts are intolerant of Bd and Ranavirus coinfection, especially at high doses. Mounting immune responses to combat these multiple deadly and rapidly replicating microparasites is costly and could limit resources that the host can allocate to other demanding processes such as growth, leading to the observed patterns (Lochmiller \& Deerenberg 2000; Greenet al. 2002; Gray et al. 2009; Kilpatrick et al.2010). However, the negative effects on host growth caused by higher loads of $\mathrm{Bd}$ seem to be mediated by higher doses of A. hamatospicula, suggesting that hosts are more tolerant to macro- and microparasite co-infections than micro- and microparasite co-infections. Additionally, these results indicate that density-dependent competition plays a role in host tolerance to these parasites and therefore host health.

All pairwise parasite comparisons were significantly impacted by dose. Additionally, the directions of the interactions were variable, with marginally positive and negative density dependent interactions. However, the strength of these interactions was highly asymmetric and always weak in one of the two directions. These results are consistent with other ecological interactions, such as interactions in food webs and in plantpollinator networks, which often show asymmetric interactions that are weak in one direction (Paine 1992; McCann et al. 1998; Bascompte et al. 2006). Asymmetric interactions have also been seen before in this co-infection system (Ramsay \& Rohr submitted). These asymmetric interactions are thought to maintain biodiversity and long-term co-existence by dampening oscillations between the interacting individuals (Paine 1992; McCann et al.1998; Bascompte et al. 2006). In this system, these patterns were likely driven by indirect interactions mediated by the host immune system and were drivers of host tolerance to these co-infections. Thus, our results suggest that density can drive parasite interactions and host health, but the direction and strength of these interactions vary. Further studies on amphibian immune systems would help better elucidate the mechanisms behind the density-dependent interactions we have found in this system.

Acknowledgements: We would like to thank J. Hoverman for supplying the Ranavirus and all the undergraduates who made this experiment possible. Funds were provided by grants to J.R.R. from the National Science Foundation (EF-1241889, IOS-1754868) and the National Institutes of Health (R01GM109499, R01TW010286-01).

\section{References:}

1. 
Abbas, A.K., Murphy, K.M. \& Sher, A. (1996). Functional diversity of helper T lymphocytes. Nature, 383, 787-793.

2 .

Ahmed, N., French, T., Rausch, S., Kuhl, A., Hemminger, K., Dunay, I.R.et al. (2017). Toxoplasma Coinfection Prevents Th2 Differentiation and Leads to a Helminth-Specific Th1 Response.Frontiers in cellular and infection microbiology, 7, 341 .

3.

Baker, M.R. (1987). Synopsis of the Nematoda Parasitic in amphibians and reptiles. Occasional papers in biology, 11 .

4.

Bascompte, J., Jordano, P. \& Olesen, J.M. (2006). Asymmetric coevolutionary networks faciliate biodiversity maintenance.Science, 312, 431-433.

5 .

Bell, A.S., de Roode, J.C., Sim, D. \& Read, A.F. (2006). Within-host compeition in genetically diverse malaria infections: parasite virulence and competitive success. Evolution , 60, 1358-1371.

6.

Berger, A. (2000). Th1 and Th2 responses: what are they? British Medical Journal , 321, 424.

7.

Berger, L., Hyatt, A.D., Speare, R. \& Longcore, J.E. (2005). Life cycle stages of the amphibian chytrid Batrachochytrium dendrobatidis .Diseases of aquatic organisms , 68, 51-63.

8.

Berger, L., Speare, R., Daszak, P., Green, D.E., Cunningham, A.A., Goggin, C.L. et al. (1998). Chytridiomycosis causes amphibian mortality associated with population declines in the rain forests of Australia and Central America. Proceedings of the National Academy of Sciences , 95.

9.

Blanco, J.L. \& Garcia, M.E. (2008). Immune response to fungal infections. Veterinary immunology and immunopathology, 125, 47-70.

10.

Boyle, D.G., Boyle, D.B., Olsen, V., Morgan, A.T. \& Hyatt, A.D. (2004). Rapid quantitative detection of chytridiomycosis (Batrachochytrium dendrobatidis ) in amphibian samples using real-time Taqman PCR assay.Diseases of aquatic organisms , 60, 141-148.

11.

Breheny, P. \& Burchett, W. (2019). visreg: Visualization of regression models, R version 2.5-1.

12.

Chesson, P. \& Kuang, J.J. (2008). The interaction between predation and competition. Nature , 456, 235-238.

13.

Cohen, J.M., Venesky, M.D., Sauer, E.L., Civitello, D.J., McMahon, T.A., Roznik, E.A. et al. (2017). The thermal mismatch hypothesis explains host susceptibility to an emerging infectious disease.Ecology letters , 20, 184-193. 
14.

Corbett, E.L., Watt, C.J., Walker, N., Maher, D., Williams, B.G., Raviglione, M.C. et al. (2003). The growing burden of Tuberculosis: global trends and interactions with the HIV epidemic.Arch Intern Med, $163,1009-1021$.

15.

de Roode, J.C., Culleton, R., Cheesman, S.J., Carter, R. \& Read, A.F. (2004). Host heterogeneity is a determinant of competitive exclusion or coexistence in genetically diverse malaria infections.Proceedings. Biological sciences , 271, 1073-1080.

16.

Degen, W.G., Daal, N., Rothwell, L., Kaiser, P. \& Schijns, V.E. (2005). Th1/Th2 polarization by viral and helminth infection in birds. Vet Microbiol , 105, 163-167.

17.

Echaubard, P., Little, K., Pauli, B. \& Lesbarreres, D. (2010). Context-dependent effects of ranaviral infection on northern leopard frog life history traits. PloS one, 5, e13723.

18.

Ezenwa, V.O. \& Jolles, A.E. (2015). Epidemiology. Opposite effects of anthelmintic treatment on microbial infection at individual versus population scales. Science, 347, 175-177.

19.

Fites, J.S. (2014). Evasion of adaptive immune defenses by the lethal chytrid fungus Batrachochytrium dendrobatidis . In:Biological Sciences . Vanderbilt University, p. 269.

20.

Fites, J.S., Reinert, L.K., Chappell, T.M. \& Rollins-Smith, L.A. (2014). Inhibition of local immune responses by the frog-killing fungus Batrachochytrium dendrobatidis. Infection and immunity, 82, 4698-4706.

21.

Gantress, J., Maniero, G.D., Cohen, N. \& Robert, J. (2003). Development and characterization of a model system to study amphibian immune responses to iridoviruses. Virology , 311, 254-262.

22.

Garner, T.W.J., Walker, S., Bosch, J., Leech, S., Marcus Rowcliffe, J., Cunningham, A.A. et al. (2009). Life history tradeoffs influence mortality associated with the amphibian pathogenBatrachochytrium dendrobatidis. Oikos , 118, 783-791.

23.

Ge, S., Zheng, D., Zhao, Y., Liu, H., Wenbo, L., Sun, Q. et al.(2012). Evaluating viral interference between Influenza virus and Newcastle disease virus using real-time reverse transcription-polymerase chain reaction in chicken eggs. Virology, 9, 1-8.

24.

Gervasi, S., Gondhalekar, C., Olson, D.H. \& Blaustein, A.R. (2013). Host identity matters in the amphibianBatrachochytrium dendrobatidis system: fine-scale patterns of variation in responses to a multi-host pathogen. PloS one, 8, e54490.

25. 
Graham, A.L. (2008). Ecological rules governing helminth-microparasite coinfection. Proceedings of the National Academy of Sciences of the United States of America , 105, 566-570.

26.

Gray, M.J., Miller, D.L. \& Hoverman, J.T. (2009). Ecology and pathology of amphibian ranaviruses. Diseases of aquatic organisms , 87, 243-266.

27.

Green, D.E., Converse, K.A. \& Schrader, A.K. (2002). Epizootiology of sixty-four amphibian morbidity and mortality events in the USA, 1996-2001. Annals New York Academy of Sciences , 969.

28.

Greub, G., Ledergerber, B., Battegay, M., Grob, P., Perrin, L., Furrer, H. et al. (2000). Clinical progression, survival, and immune recovery during antiretroviral therapy in patients with HIV-1 and hepatitis C virus coinfection: the Swiss HIV Cohort Study. The Lancet , 356, 1800-1805.

29.

Hothorn, W. (2010). multcomp: simultaneous inference in general parametric models, R package version $1.4-10$.

30.

Hoverman, J.T., Gray, M.J. \& Miller, D.L. (2010). Anuran susceptibilities to ranaviruses: role of species identity, exposure route, and a novel virus isolate. Diseases of aquatic organisms , 89, 97-107.

31.

Hoverman, J.T., Mihaljevic, J.R., Richgels, K.L., Kerby, J.L. \& Johnson, P.T. (2012). Widespread cooccurrence of virulent pathogens within California amphibian communities. Ecohealth , 9, 288-292.

32.

Jamieson, A.M., Pasman, L., Yu, S., Gamradt, P., Homer, R.J., Decker, T.et al. (2013). Role of tissue protection in lethal respiratory viral-bacterial coinfection. Science, 340, 1230-1234.

33.

Johnson, P.T., de Roode, J.C. \& Fenton, A. (2015). Why infectious disease research needs community ecology. Science, 349, 1259504.

34.

Johnson, P.T.J. \& Buller, I.D. (2011). Parasite competition hidden by correlated coinfection: using surveys and experiments to understand parasite interactions. Ecology , 92, 535-541.

35.

Kassambara, A., Kosinski, M., Biecek, P. \& Fabian, S. (2019). Drawing survival curves using 'ggplot2'. $R$ package version 0.4 .6 .

36.

Khuroo, M.S. (1996). Ascariasis. Gastroenterology clinics of North America, 25, 553-577.

37.

Kilpatrick, A.M., Briggs, C.J. \& Daszak, P. (2010). The ecology and impact of chytridiomycosis: an emerging disease of amphibians. Trends in ecology \& evolution , 25, 109-118.

38. 
Kim, J., Chung, H.K. \& Chae, C. (2003). Association of porcine circovirus 2 with porcine respiratory disease complex. The Veterinary Journal , 166, 251-256.

39.

Knutie, S.A., Shea, L.A., Kupselaitis, M., Wilkinson, C.L., Kohl, K.D. \& Rohr, J.R. (2017a). Early-Life Diet Affects Host Microbiota and Later-Life Defenses Against Parasites in Frogs. Integrative and comparative biology , 57, 732-742.

40.

Knutie, S.A., Wilkinson, C.L., Wu, Q.C., Ortega, C.N. \& Rohr, J.R. (2017b). Host resistance and tolerance of parasitic gut worms depend on resource availability. Oecologia , 183, 1031-1040.

41.

Kuris, A.M. \& Lafferty, K.D. (1994). Community structure: larval trematodes in snail hosts. Annual Review of Ecology and Systematics , 25, 189-217.

42.

Lochmiller, R.L. \& Deerenberg, C. (2000). Trade-offs in evolutionary immunology: just what is the cost of immunity? Oikos , 88, 87-98.

43.

McCann, K., Hastings, A. \& Huxel, G.R. (1998). Weak trophic interactions and the balance of nature. Nature , 395, 794-798.

44.

McMahon, T.A., Sears, B.F., Venesky, M.D., Bessler, S.M., Brown, J.M., Deutsch, K. et al. (2014). Amphibians acquire resistance to live and dead fungus overcoming fungal immunosuppression. Nature, 511, 224-227.

45.

Morel, P.A. \& Oriss, T.B. (1998). Crossregulation between Th1 and Th2 cells. Critical reviews in immunology , 18, 275-303.

46.

Motran, C.C., Silvane, L., Chiapello, L.S., Theumer, M.G., Ambrosio, L.F., Volpini, X. et al. (2018). Helminth Infections: Recognition and Modulation of the Immune Response by Innate Immune Cells. Front Immunol , 9,664 .

47.

Niczyporuk, J.S., Woźniakowski, G., Czekaj, H. \& Samorek-Salamonowicz, E. (2014). Interactions between Marek's disease virus Rispens/CVI988 vaccine strain and adenovirus field strain in chicken embryo fibroblast (CEF) cultures. Polish Journal of Veterinary Sciences , 17, 3-8.

48.

Ortega, N., Price, W., Campbell, T. \& Rohr, J. (2015). Acquired and introduced macroparasites of the invasive Cuban treefrog, Osteopilus septentrionalis. International journal for parasitology. Parasites and wildlife , 4, 379-384.

49.

Paine, R.T. (1992). Food-web analysis through field measurement of per capita interaction strength. Nature , 355, 73-75. 
50.

Paul, W.E. \& Zhu, J. (2010). How are T(H)2-type immune responses initiated and amplified? Nature reviews. Immunology, 10, 225-235.

51.

Pearman, P.B., Garner, T.W., Straub, M. \& Greber, U.F. (2004). Response of the Italian agile frog (Rana latastei) to a Ranavirus, frog virus 3: a model for viral emergence in naive populations. Journal of wildlife diseases , 40, 660-669.

52.

Pedersen, A.B. \& Fenton, A. (2007). Emphasizing the ecology in parasite community ecology. Trends in ecology $\&$ evolution, 22, 133-139.

53.

Picco, A.M., Brunner, J.L. \& Collins, J.P. (2007). Susceptibility of the endangered California tiger salamander, Ambystoma californiense, to ranavirus infection. Journal of wildlife diseases , 43, 286-290.

54.

R Core Team (2019). R: a language and environement for statistical computing. . In: $R$ Foundation for Statistical Computing, Vienna, Austria .

55.

Ramsay, C. \& Rohr, J.R. (submitted). The application of community ecology theory to co-infections in wildlife hosts. Ecology .

56.

Rojas, S., Richards, K., Jancovich, J.K. \& Davidson, E.W. (2005). Influence of temperature on Ranavirus infection in larval salamanders Ambystoma tigrinum. Diseases of aquatic organisms , 63, 95-100.

57.

Rosenblum, E.B., Poorten, T.J., Settles, M. \& Murdoch, G.K. (2012). Only skin deep: shared genetic response to the deadly chytrid fungus in susceptible frog species. Molecular ecology, 21, 3110-3120.

58.

Salgame, P., Yap, G.S. \& Gause, W.C. (2013). Effect of helminth-induced immunity on infections with microbial pathogens. Nat Immunol , 14, 1118-1126.

59.

Skerratt, L.F., Berger, L., Speare, R., Cashins, S., McDonald, K.R., Phillott, A.D. et al. (2007). Spread of Chytridiomycosis Has Caused the Rapid Global Decline and Extinction of Frogs.EcoHealth , 4, 125-134.

60.

Stutz, W.E., Blaustein, A.R., Briggs, C.J., Hoverman, J.T., Rohr, J.R. \& Johnson, P.T.J. (2018). Using multi-response models to investigate pathogen coinfections across scales: insights from emerging diseases of amphibians. Methods Ecol Evol , 9, 1109-1120.

61.

Swanson, S.J., Neitzel, D., Reed, K.D. \& Belongia, E.A. (2006). Coinfections acquired from ixodes ticks. Clin Microbiol Rev , 19, 708-727.

62. 
Therneau, T.M. \& Lumley, T. (2019). survival: Survival analysis, R version 2.44-1.1.

63.

Vhora, M.S. \& Bolek, M.G. (2013). New host and distribution records for Aplectana hamatospicula (Ascaridida: Cosmocercidae) in Gastrophryne olivacea (Anura: Microhylidae) from the Great Plains U.S.A. The Journal of parasitology, 99, 417-420.

64.

Voyles, J., Young, S., Berger, L., Campbell, C., Voyles, W.F., Dinudom, A. et al. (2009). Pathogenesis of Chytridiomycosis, a cause of catastrophic amphibian declines. Science , 326, 582-585.

65.

Wake, D.B. \& Vredenburg, V.T. (2008). Colloquium paper: are we in the midst of the sixth mass extinction? A view from the world of amphibians.Proceedings of the National Academy of Sciences of the United States of America , 105 Suppl 1, 11466-11473.

66.

Warne, R.W., LaBumbard, B., LaGrange, S., Vredenburg, V.T. \& Catenazzi, A. (2016). Co-Infection by Chytrid Fungus and Ranaviruses in Wild and Harvested Frogs in the Tropical Andes. PloS one , 11, e0145864.

67.

Watters, J.L., Davis, D.R., Yuri, T. \& Siler, C.D. (2018). Concurrent Infection of Batrachochytrium dendrobatidis and Ranavirus among Native Amphibians from Northeastern Oklahoma, USA. J Aquat Anim Health , 30, 291-301.

68.

Yazdanbakhsh, M., Kremsner, P.G. \& van Ree, R. (2002). Allergy, Parasites, and the Hygiene Hypothesis. Science, 296, 490-494.

69.

Zelante, T., De Luca, A., D'Angelo, C., Moretti, S. \& Romani, L. (2009). IL-17/Th17 in anti-fungal immunity: what's new? Eur J Immunol , 39, 645-648.

Table 1 . Hosts were co-exposed to one parasite at a fixed density and a second parasite over a range of densities shown below.

\begin{tabular}{lllll}
\hline Parasite & \multicolumn{5}{l}{ Doses } \\
\hline Ranavirus (PFUs) & $1.00 \mathrm{E}+03$ & $1.00 \mathrm{E}+04$ & $4.50 \mathrm{E}+04$ & $1.00 \mathrm{E}+05$ \\
Bd (zoospores) & $1.00 \mathrm{E}+03$ & $1.00 \mathrm{E}+04$ & $4.50 \mathrm{E}+04$ & $1.00 \mathrm{E}+05$ \\
A. hamatospicula (J3 larvae) & 15 & 30 & 45 & 60 \\
\hline
\end{tabular}

Fig. 1. The proportional dose of either Batrachochytrium dendrobatidis or Aplectana hamatospicula (there was no difference between the two) was negatively related to load of Ranavirus on Osteopilus septentrionalis hosts $\left(\chi^{2}{ }_{1}=4.07, p=0.04\right)$. Dose of co-infecting parasite is provided as a proportion of the maximum dose such that 1.0 is the highest dose and 0.0 is a host singly infected with Ranavirus. Shown is shown as a conditional plot displaying the expected value (dashed line), a confidence interval for the expected value (gray band), and partial residuals (points).

Fig. 2 . The dose of Batrachochytrium dendrobatidis (Bd) was negatively associated with Aplectana hamatospicula load $(p<0.001)$, whereas the dose of Ranavirus $(\mathrm{Rv})$ was not significantly associated with A. 
hamatospicula load ( $p=0.22$; Dose $\mathrm{x}$ parasite identity: $\left.\chi^{2}{ }_{1}=17.2, p<0.001\right)$. Dose of co-infecting parasite is provided as a proportion of the maximum dose. Shown are conditional plots displaying the expected value (dashed line), a confidence interval for the expected value (gray band), and partial residuals (points).

Fig. 3. All three parasites reduced host growth rate relative to hosts that were not infected, but Bactrachochytrium dendrobatidis (Bd) was the most virulent of the parasites, reducing host growth significantly more than Aplectana hamatospicula (Ah) or Ranavirus (Rv). Shown are conditional plots displaying the expected value (dashed line), a confidence interval for the expected value (gray band), and partial residuals (points). Treatments that share letters are not different from one another based on a Tukey HSD multiple comparison test.

Fig. 4. Hosts exposed to Bactrochochytrium dendrobatidis(Bd) as the fixed-dose parasite had significantly reduced survival relative to hosts exposed to Aplectana hamatospicula (Ah; $p<0.001$ ), Ranavirus (Rv; $p$ $<0.001$ ) or no parasite (Control; $p<0.001$ ), regardless of the identity or dose of the changing dose parasite. Survival curves shown as median survival (solid line) with the associated $95 \%$ confidence interval (grey band).

Fig. 5. Tolerance (defined as less negative slopes between parasite load and growth rate) of Batrachochytrium dendrobatidis $(\mathrm{Bd})$ was associated negatively with the dose of Ranavirus $(\mathrm{Rv}, \mathbf{A})$, but positively with the dose of Aplectana hamatospicula (Ah, B ). Shown are the conditional plots displaying the expected value (dashed line), a confidence interval for the expected value (gray band), and partial residuals (points).

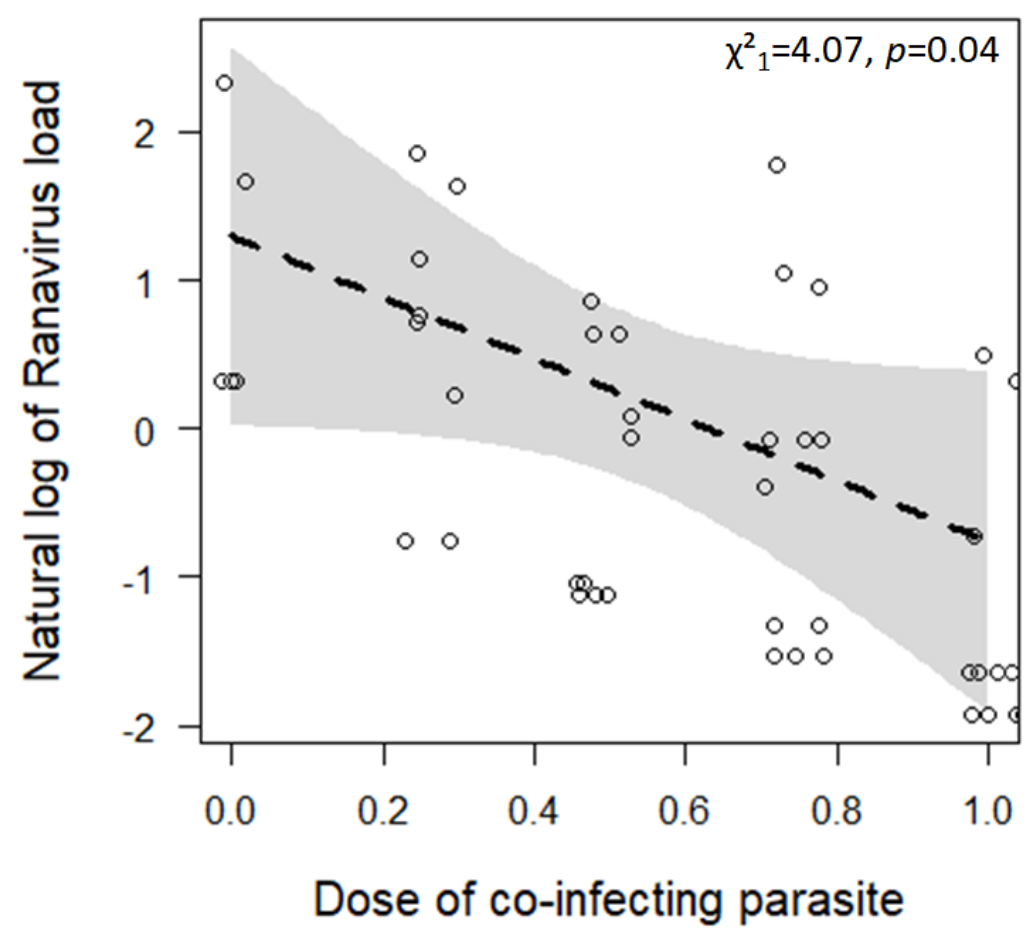

Fig. 1 . 


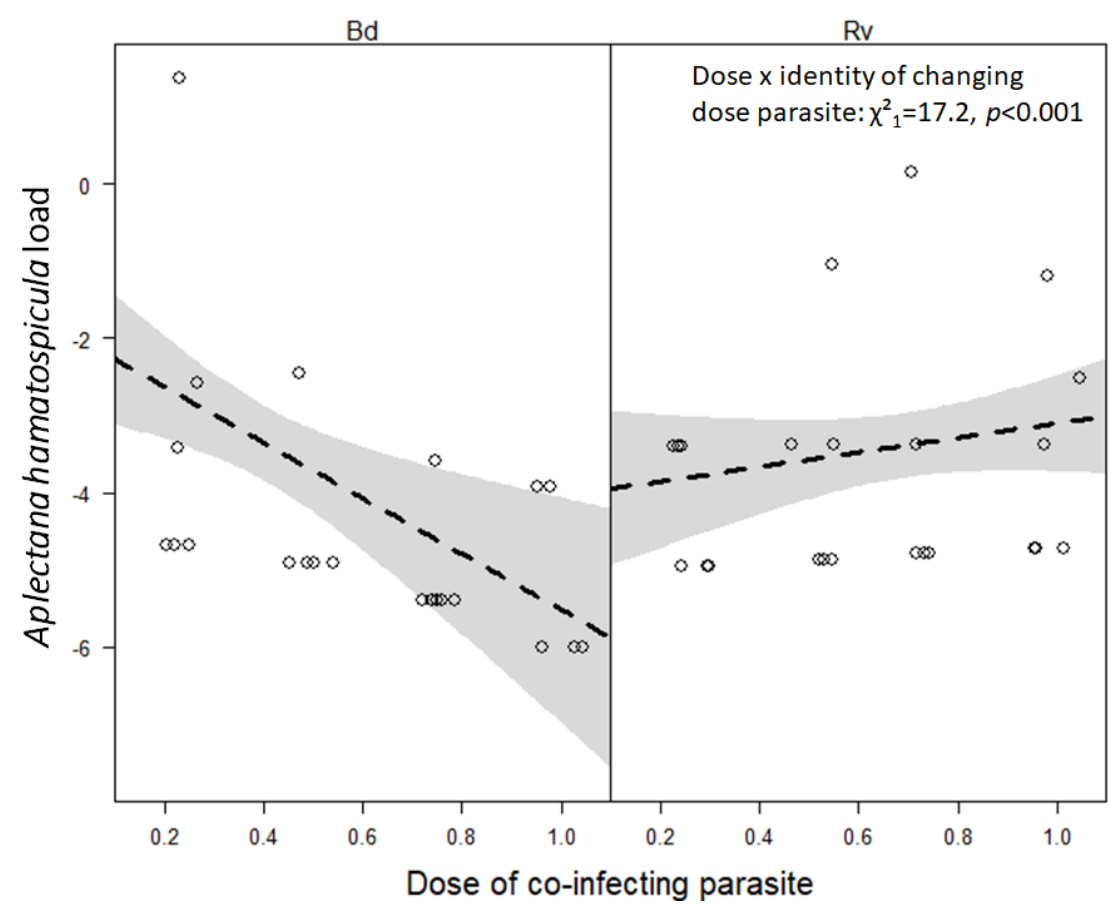

Fig. 2 .

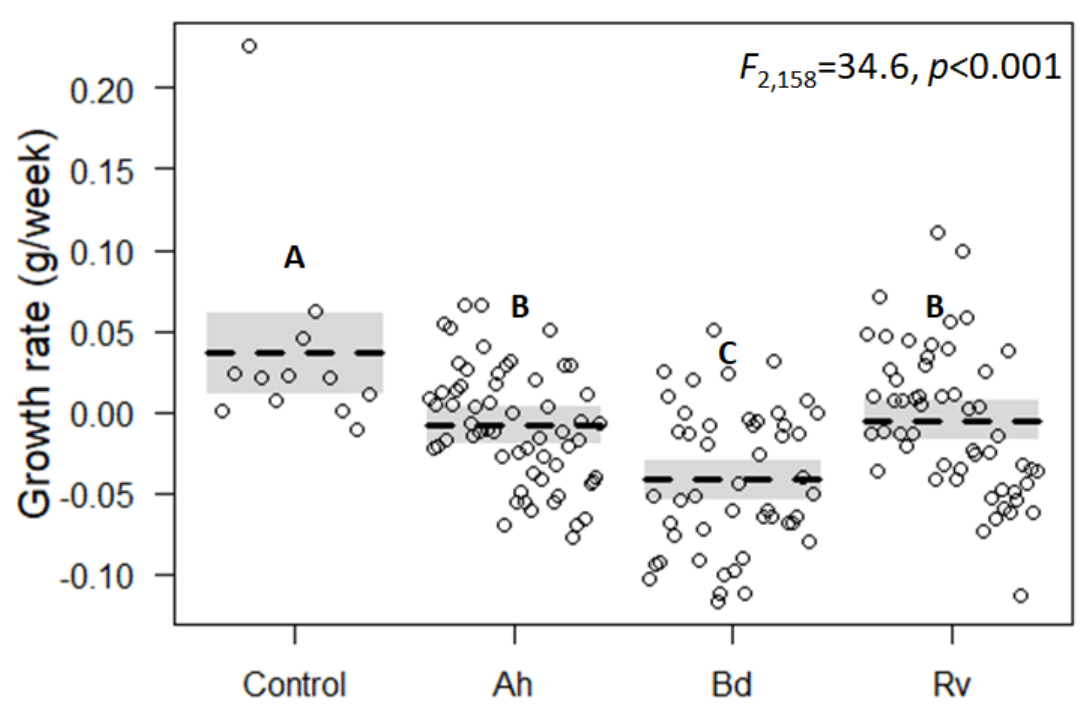

Fixed-dose parasite

Fig. 3. 


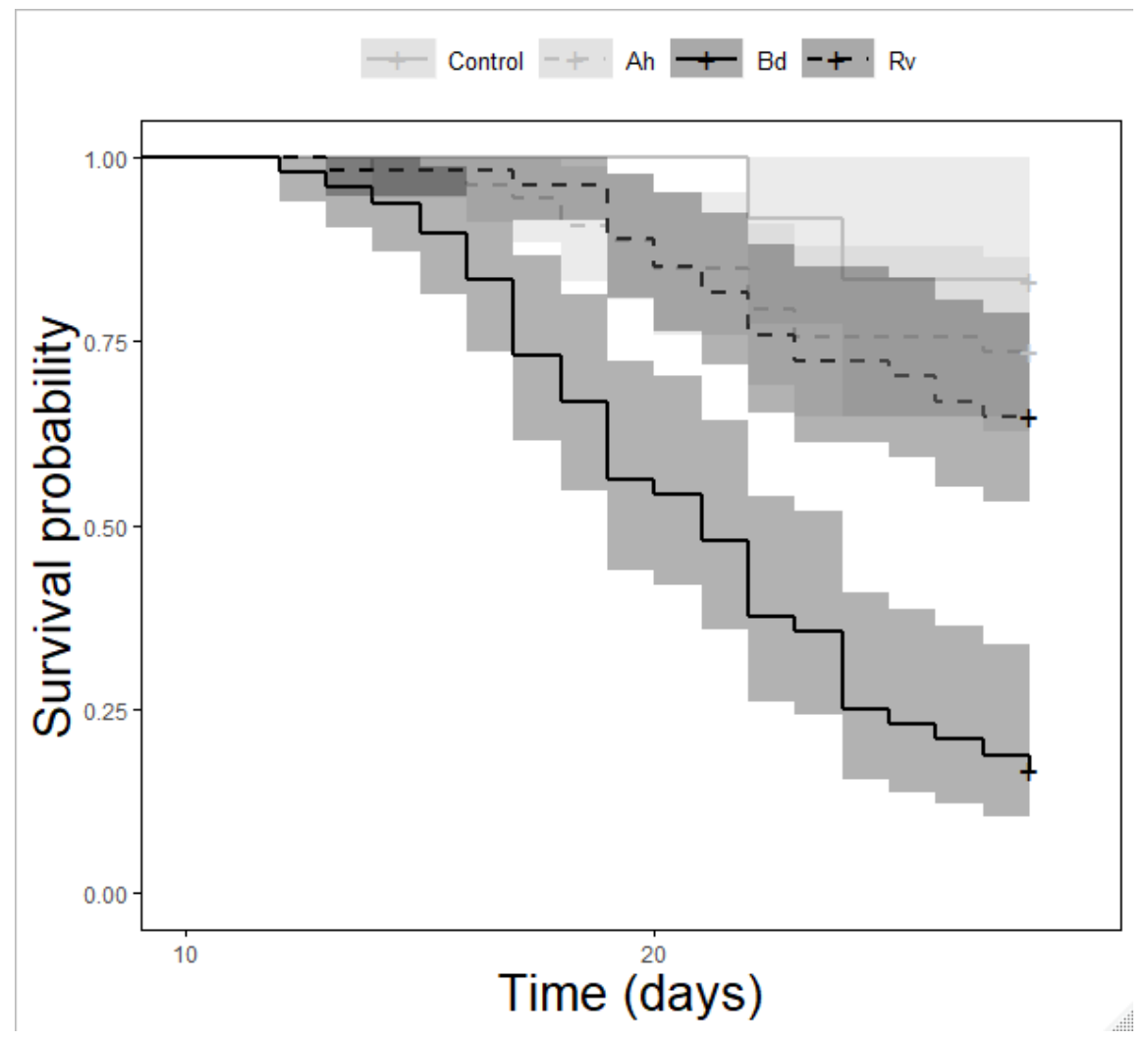

Fig. 4. 
A
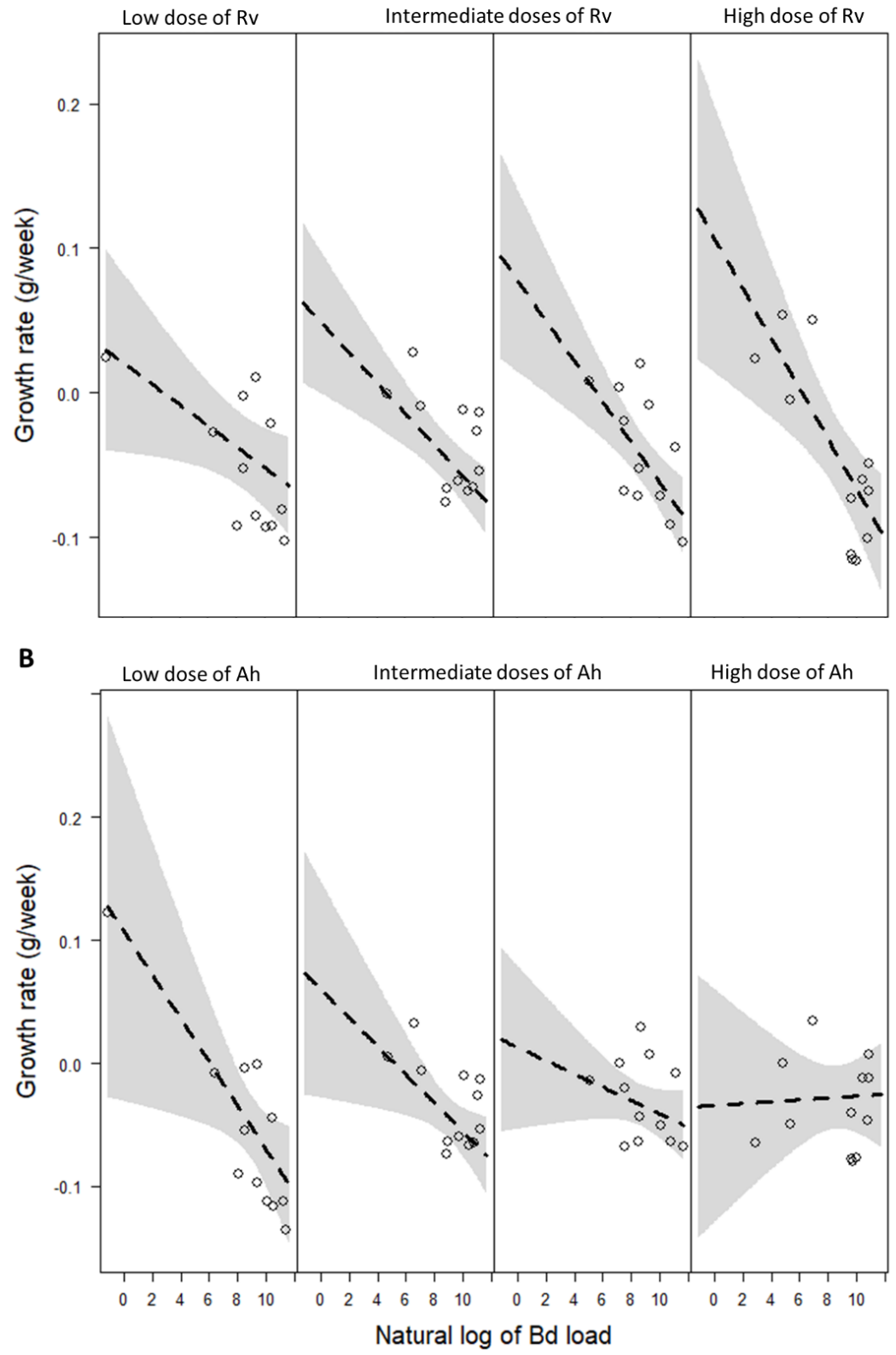

Fig. 5 . 